\title{
Recent Development in In situ Ion Irradiation Transmission Electron Microscopy
}

\author{
K. Hattar ${ }^{1}$, S.M. Hoppe ${ }^{1}$, C.Chisholm ${ }^{2}$, O.J. El-Atwani ${ }^{3}$, B.A. Hernandez-Sanchez ${ }^{1}$, H. Bei ${ }^{4}$, E.P. \\ George $^{4}$, P. Hosemann ${ }^{5}$, and A.M. Minor ${ }^{2}$ \\ 1. Sandia National Laboratories, Albuquerque, NM, USA. \\ 2. Department of Material Science and Engineering, University of California, Berkeley and National \\ Center for Electron Microscopy, Lawrence Berkeley National Laboratory, Berkeley, CA, USA. \\ 3. School of Nuclear Engineering, Purdue University, West Lafayette, IN, USA \\ 4. Materials Science and Technology Division, Oak Ridge National Laboratory, Oak Ridge, TN, USA \\ 5. Department of Nuclear Engineering, University of California, Berkeley, CA, USA.
}

In order to understand the structural response of materials at the nanoscale to various extreme environments, an effort has been made since nearly the inception of transmission electron microscopy (TEM) to simulate these environments within the microscope. To simulate radiation environments in the TEM, microscopists have capitalized on the original serendipitous observation of dislocation loop formation from in situ oxygen ion irradiation from a contaminated tungsten filament made by Pashley and Presland in 1961 [1]. In the first 50 years of transmission electron microscopes with in situ ion irradiation capabilities, a total of 31 facilities around the world have been publicly demonstrated with 11 known to be operating in 2011 [2]. Recent advancements occurring in both TEM and ion beam line technology are subsequently incorporated into the new in situ TEM ion irradiation facilities providing ever increasing ion beam control, TEM resolution, analytical capability, and in situ capabilities.

The newest in situ ion irradiation TEM ( $\left.\mathrm{I}^{3} \mathrm{TEM}\right)$ facility to be brought into operation is at Sandia National Laboratories' new Ion Beam Lab that contains five accelerators and custom end stations for in situ optical, electron, and ion microscopy studies. The I ${ }^{3} \mathrm{TEM}$ is a combination of a JEOL $2100 \mathrm{LaB}_{6}$ TEM with a $6 \mathrm{MV}$ Tandem Accelerator and a $10 \mathrm{kV}$ Colutron that provides adequate ion beam characterization and control, pumping capability, as well as vibration and electrical isolation of the three systems (Figure 1A). Recent real time videos of experiments will be presented in which the combination of heavy ion irradiation and light ion implantation were applied to a well-characterized severe plastically deformed tungsten sample [3]. This facility also permits enhanced three-dimensional data collection with a high-tilt pole piece, automated software, and a high-tilt TEM stage (Figure 1B). This presentation will highlight some of the initial work done to create four-dimensional reconstructions of both gold nanoparticles and tungstate nanoscintillators, as a function of ion dose and ion species [4]. Other advanced capabilities that achieved with the I ${ }^{3}$ TEM incorporate the combination of in situ ion irradiation and quantitative small scale mechanical property testing (Figure 1C). With this technique, Mo-alloy nanofibers [5] were irradiated in situ with $3 \mathrm{MeV} \mathrm{Cu}$ to a dose of approximately 1 x $10^{7}$ and then strained to failure using a Hysitron "push-to-pull" (PTP) device. Initial results from these studies will be discussed and demonstrate the significant potential for the combination of in situ ion irradiation and quantitative small scale mechanical testing (Figure 2). Finally, a discussion on the continued development of the I ${ }^{3}$ TEM facility at Sandia National Laboratories and the possible future directions for transmission electron microscopes with in situ ion irradiation capabilities will be presented [6].

References:

[1] DW Pashley and AEB Presland, Philos. Mag., 6 (1961), p.1003

[2] JA Hinks, NIMB 267 (2009), p. 3652-3663. 
[3] O El-Atwani,M Efe, B Heim and JP Allain, JNM 434 (2013), p.170.

[4] SM Hoppe et al, Proceeding of SPIE 8509 (2012)

[5] C Chisholm et al, Acta Mater, 60 (2012), p. 2258-2264

[6] This work was partially supported by the Department of Energy's Office of Basic Energy Sciences (KH and SMH) and the Center for Defect Physics, an Energy Frontier Research Center funded by the U.S. Department of Energy's Office of Basic Energy Sciences (CC, HB, EPG, and AMM). Sandia National Laboratories is a multi-program laboratory managed and operated by Sandia Corporation, a wholly owned subsidiary of Lockheed Martin Corporation, for the U.S. Department of Energy's National Nuclear Security Administration under contract DE-AC04-94AL85000.

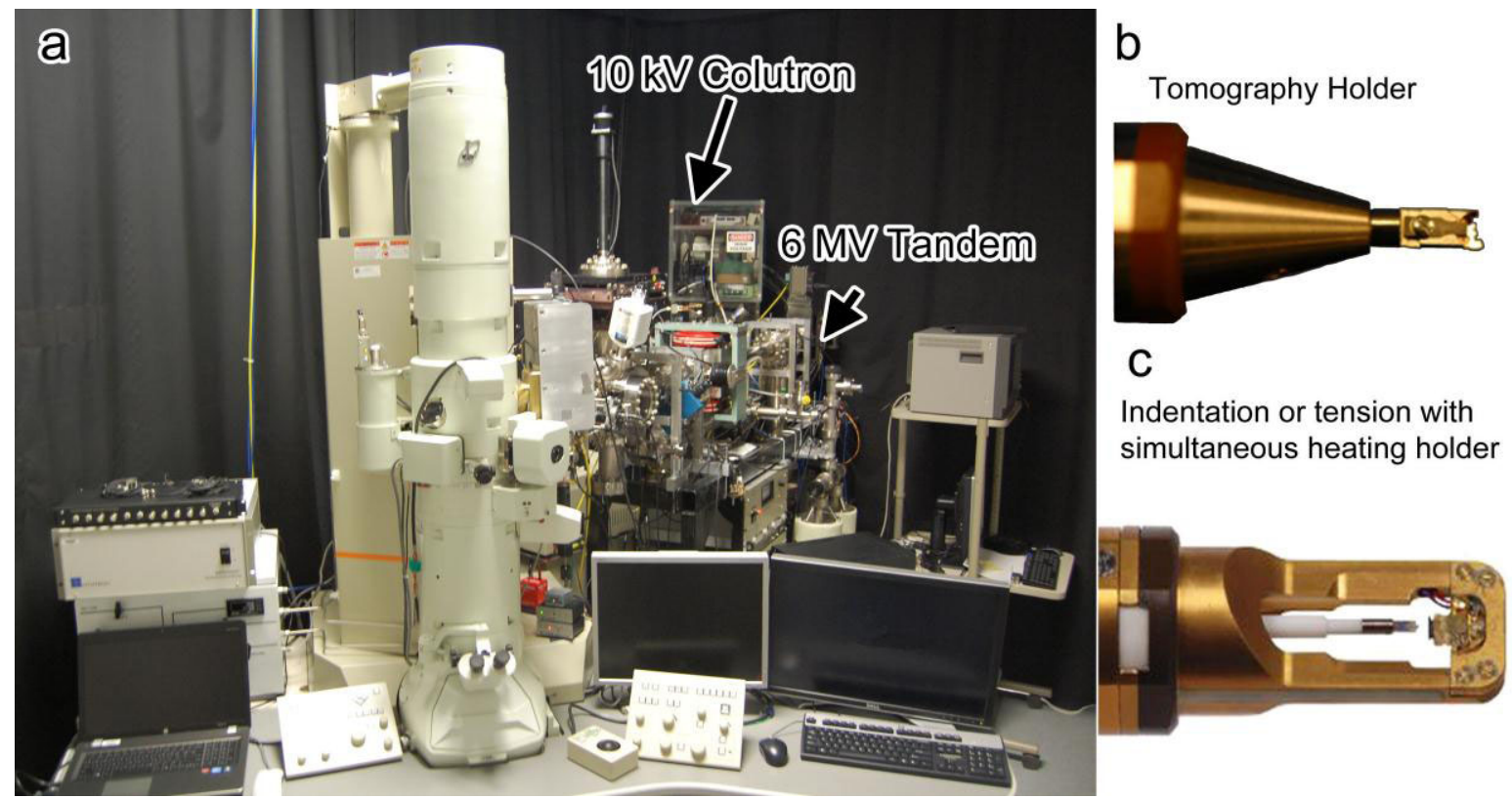

Figure 1. A) Sandia National Laboratories' in situ ion irradiation TEM. B) Hummingbird high-tilt stage for 4D reconstructions. C) Hysitron PI-95 stage for quantitative small-scale mechanical testing.
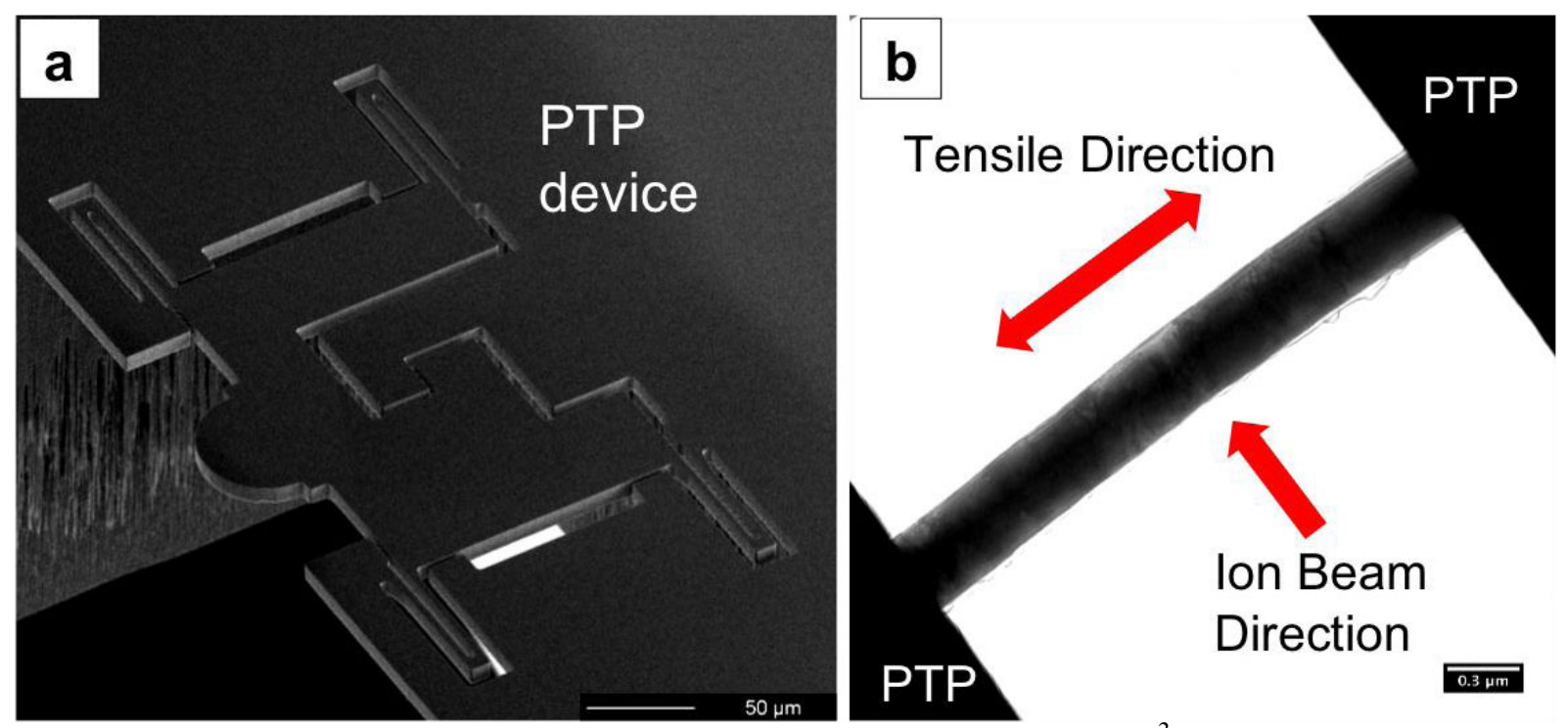

Figure 2. A) SEM micrograph of the Push-to-Pull device operated in the I ${ }^{3}$ TEM B) TEM image of Moalloy tensile sample in the experimental setup. 